\title{
El hipertexto en la divulgación científica: análisis de su uso en el perfil de twitter @materia_ciencia de El País como caso objeto de estudio
}

\author{
Aida María de Vicente Domínguez \\ Universidad de Málaga \\ aidamaria@uma.es \\ https://orcid.org/0000-0002-7733-9366
}

\author{
Nereida Cea Esteruelas \\ Universitat de Málaga \\ nereidacea@uma.es \\ https://orcid.org/0000-0002-8085-5097
}

\section{Hypertext in Scientific Disclosure: Analysis of its Use on the Twitter profile @materia-ciencia of el País as the Subject of Study \\ RESUMEN ABSTRACT}

Se investiga el uso del hipertexto para documentar o contextualizar los acontecimientos científicos publicados en

el twitter @materia_ciencia de El País. Los objetivos del estudio son: investigar la calidad de los hiperenlaces según la media de enlaces insertados; identificar las tipologías de

hiperenlaces usadas; describir las modalidades que conforman cada una de las tipologías; indicar las modalidades más usadas en cada tipología. La metodología se basa en analizar las informaciones publicadas durante un trimestre

(marzo-mayo de 2019). Los resultados indican una calidad muy alta de este recurso documental usando principalmente dos tipologías: los cronológicos y los documentales. Las conclusiones aportan una primera clasificación conceptual de los hipertextos para divulgar la ciencia en los medios de comunicación conformada por 6 modalidades: curriculares,

metodológico-científicos, organizacionales; bibliográficos; archivados y terminológicos. Asimismo, se propone un modelo de construcción discursivo multilineal basado en la conexión de textos digitalizados para divulgar los hallazgos científico-tecnológicos en los medios de utilidad para el sector académico o profesional, y como base metodológica para

futuros estudios, siendo transversal a otras disciplinas.

PALABRAS CLAVE

Divulgación científica; Documentación; Enlaces semánticos; Hipertexto; Redes sociales; Medios de comunicación
The use of hypertext to document or contextualize scientific events published on Twitter amateria-ciencia of El Pais is investigated. The objectives of the study are: to investigate the quality according to the average number of inserted links; to identify the typologies used; to describe the modality that forms each one of the typologies and to indicate the most used modalities in each typology. The methodology is based on analysing the information published during one term ( March-May 2019). The results indicate this documentary resource is of very quality using mainly two typologies: the chronological and the documentary. The conclusions provide the first conceptual classification of useful hypertexts to disclose science in the media made up of six modalities: curricular, scientific methodological, organizational, bibliographic, archived, terminological. In addition, a model of construction of multilineal discursive based on the connection of digitalized texts to disclose useful scientific-technological findings is proposed, for the professional academic sector, and a methodological base for future studies, being transversal to other disciplines.

KEYWORDS

Scientific disclosure; Documentation; Semantic links; Hypertexts; Social networks; Media 


\section{Introducción}

El periodismo científico "especialización informativa que consiste en divulgar la ciencia y la tecnología a través de los medios de comunicación de masas" (Calvo Hernando, 1992, p. 22) es una profesión que se rige por unas pautas específicas cuyo conocimiento es primordial para informar con exhaustividad y también con mucha claridad a un público que, sin ser experto en la materia, pueda comprender e interiorizar la información sin realizar un doble esfuerzo de lectura. Un público que como indica Lionnais debe abarcar desde "la escuela maternal al premio nobel porque hay que divulgar a todos esos niveles" (Belenguer, 2003, p. 45- 46).

Para conseguir este fin, es preciso dominar estrategias de comprensión lectora o recursos para documentar la información y el hipertexto es un recurso documental apto. Y es que "en el caso de la noticia científica, es forzoso situarla dentro del contexto generalizado de las sociedades humanas en sus respectivas civilizaciones" (Calvo, 1990, p. 99). Lo que hace necesario que en la pieza informativa se explique, precise y aclare, el sentido, los antecedentes y consecuencias de un descubrimiento. Al respecto, Diezhandino (1997) insistía: "además de describir los hechos básicos que permitan entender nuestro mundo y lo que esos hechos significan, hay que indicar por qué son importantes, en qué contexto hay que situarlos, a dónde nos conducen." (p. 87). Y en este estudio se pretende abordar cómo se hace uso de los enlaces para contextualizar los acontecimientos científicos en una edición digital.

Una investigación considerada relevante porque el hiperenlace en la información científica no se ha investigado. Estudios precedentes han analizado los hipertextos en los medios colombianos (Llano, 2005; Fondevila y Segura, 2012); los dos principales periódicos digitales de Colombia y de sus países fronterizos territoriales de habla hispana: Venezuela, Ecuador, Perú y Panamá (Castellanos, 2011); en el diario Clarín (Argentina), Le Monde (Francia), USA Today (EE.UU) o The Telegraph de Reino Unido (Fondevila, Beriain, Perelló y Barbero, 2014); los medios eslovenos (Oblack, 2005); su implementación en la Gaceta de Galicia de la Facultad de Ciencias de la Comunicación de la Universidad de Santiago de Compostela (López, Pereira y Gage, 2001); y en los medios de comunicación españoles se ha investigado el uso de los enlaces en la prensa deportiva (Fondevila, Beriain, del Olmo y Valero, 2014); realizado análisis comparativos entre la prensa deportiva española y francesa (Fondevila, Rom y Santana, 2016); y en los medios generalistas españoles (Barredo, 2003; Pérez Marco, 2001; Bernandino y Cebrian, 2017; Arias-Robles y García_Avilés, 2018; Orero, Bernandino y Cebrian, 2019).

En este trabajo se pretende aportar nuevos datos a esta línea de investigación analizando cómo se usan los enlaces para contextualizar los hechos científico-tecnológicos a una audiencia generalista. En concreto, los estudios realizados sobre los medios generalistas citados analizaron el uso de los enlaces en los contenidos alusivos a la figura del rey Juan Carlos, (Barredo, 2003); su uso en la sección internacional, nacional, sociedad, cultura, economía y deportes, excepto en la sección ciencia, (Pérez Marco, 2001). O bien, se centraron en analizar las portadas de los diarios generalistas nacionales (Oreo y Bernandino, 2017; Orero, Bernandino y Cebrian, 2019). Ahora, se investiga la hipertextualidad en los enlaces de las piezas informativas que conforman una sección específica sobre ciencia en el perfil social de twitter @amateria_ciencia de El País

Significativo es también que hasta el momento se han recomendado diversos recursos para contextualizar o documentar los hechos científico- tecnológicos que ayuden a una lectura comprensiva del acontecimiento. Tales como la metáfora "para generar asociaciones e implicaciones entre los fenómenos ya conocidos y aquellos nuevos que pretenden explicar" (Galán, 2001, p. 129) y que hasta el propio Einstein no desdeñaba en su uso "para comprender, de forma intuitiva, la identidad de la masa inerte y la masa pesante, o el hecho de que los sucesos simultáneos sólo lo son con respecto a un mismo sistema" (Calvo, 1990, p. 70). Otros recursos son los símiles, los destacados, las anécdotas o los ejemplos cercanos. No obstante, no hay referencias sobre el uso del hipertexto en la divulgación científica. Y "Los enlaces hipertextuales son uno de los grandes conjuntos de estrategias distintivas de la narrativa digital (Salaverría, 1999; Armentia, Caminos, Elexgaray et al., 2000a, 2000b; Rost, 2003; Oblak, 2005; Larrondo, 2009a, 2009b, 2010; Rubio y Blanco, 2010; Steensen, 2011)" (Barredo, 2003, p. 89).

En este contexto, se pretende conocer cómo se usa el hipertexto para contextualizar estos hechos en los medios de comunicación generalistas españoles para divulgar la ciencia, entendiendo por hipertexto "un contenido informativo constituido por una nebulosa de fragmentos, donde el sentido se construye a través de cada uno de los caminos de lectura" (Balpe 1990, citado en Borrás, 2005, p. 34) o "un texto que contiene elementos o documentos entrelazados a partir de los cuales se puede acceder a otra información" (García-García, Arévalo y Hernández, 2019, p. 290) y en el que el usuario "puede tener la posibilidad de profundizar en el contenido de la noticia en la medida que lo necesite" (López, Pereira y Gage, 2001, p. 112). En concreto, se pretenden analizar los hipertextos semánticos "es decir, lo que relacionan directamente los propios contenidos entre sí [...] para generar sentido, asociar contenidos, más allá de la estructuración rígida e inamovible que imprime categorías" (Rost, 2003, p. 176), pero exclusivamente los que conformen la pieza informativa (título, lead y cuerpo de la información).

Unos datos de interés porque aportan al campo académico y profesional datos sobre cómo usarlos para elaborar textos claros y entendibles por una audiencia generalista. Como indica Díaz Noci (2018) es "necesario determinar no sólo cuál 
es la sintaxis hipertextual de las noticias, sino también cuáles son las estructuras más habituales, para así establecer si se manifiestan determinados patrones de uso, cuáles son y la razón por la que se producen" (p. 231).

Pautas muy necesarias en el sector o sección analizada para eliminar las barreras, principalmente, entre la ciencia y la sociedad. Y también porque de su explicación depende la orientación de la opinión pública, por lo cual esa función tiene una considerable importancia, principalmente como señala Rodrigo (1989) ante "el carácter problemático, ambiguo o ambivalente de determinados acontecimientos" (p. 164). Por ello, el principal esfuerzo del divulgador científico está en buscar la mejor forma de explicar el hecho para acercarlo al lector. Para este fin "la documentación es pieza clave en los textos periodísticos del periodismo de explicación y, por extensión, del periodismo especializado" (Herrero, 1997, p. 73), y el hipertexto como recurso documental aporta esta significación, al tiempo que ayuda a elevar el nivel de conocimiento del lector fomentado la cultura científica en la sociedad.

Asimismo, ofrece la posibilidad de enlazar con fuentes originales de la información dada. Es un recurso que permite, la transparencia y el acceso directo a los datos, dos valores que la cultura digital en red es capaz de fomentar, sobre todo, por medio de la hipertextualidad. Lo que aporta mayor credibilidad a la información. Al respecto, De Maeyer (2012) considera que los enlaces hacen a las noticias más creíbles al dirigir directamente al lector a más información de fondo, a más contexto, hechos y fuentes.

\section{Objetivos y metodología}

Los objetivos de la investigación son los siguientes:

- Averiguar la calidad de los hipervínculos según el número de inserciones en la pieza informativa.

- Identificar las tipologías de enlaces usados para divulgar la ciencia.

- Describir las diferentes modalidades que conforman cada tipología.

- Desvelar cuáles son las modalidades más representativas.

Y para ello se establece una metodología estructurada en las siguientes fases:

1. Investigación bibliográfica y documental en diversas bases de datos nacionales e internacionales (Dialnet, Teseo, Google Scholar y B-on) a través de las siguientes ecuaciones de búsqueda: hipertexto, hipervínculos, hipertextualidad, periodismo, prensa digital, prensa española, ciencia y divulgación científica. Palabras claves que se combinan en la pesquisa para reducir la pérdida de información.

2. La selección de los medios, al enfocarse la investigación en los medios generalistas españoles, se opta por escoger el que tenga mayor tirada y difusión según la oficina de la Justificación de la difusión (OJD); el ranking Alexa y comScore. Todas estas dedicadas al estudio de las audiencias en España. Y el diario resultante es El País.

3. La selección de la muestra tomando como referencia la tesis doctoral de Pérez Marco (2003) se basa en una acotación temporal de tres meses (en este caso marzo, abril y mayo de 2019) y posterior selección al azar de una muestra aleatoria estratificada. En dicha tesis doctoral la acotación temporal fue de 15 días escogiendo dos semanas al azar, y entre éstas, 3 días por semana eliminado el séptimo día o último tomando como base la metodología de la semana compuesta o semana construida (Stempel y Westley, 1989). Si bien, en este estudio se amplía la muestra a tres semanas, una por mes escogidas al azar, y se computan en su totalidad ( $\mathrm{n}=245)$.

4. Las piezas informativas que conforman la unidad de análisis se toman del perfil en Twitter oficial materia @materia_ciencia: la web de noticias de ciencia, tecnología, salud y medio ambiente del País, porque la hemeroteca digital no permite seleccionar las piezas por sección y acotación temporal. Las semanas objeto de estudio son; del 11 al 17 de marzo, de 27 al 31 de mayo, y del 10 al 17 de junio. Y se excluyen del análisis aquellas que cumplen alguno de los siguientes criterios: retuiteadas; repetidas; son un volcado de la edición en papel; o elaboradas por el medio para otros organismos.

5. Para investigar si el medio usa enlaces para divulgar la ciencia se aplica un sistema binomial (SI/NO) a cada una de las piezas informativas objeto de estudio para computar su presencia o ausencia en la muestra analizada. Y para calcular la media de enlaces se toma como referencia la metodología aplicada por Barredo (2003, p. 95) denominada variable de detección para codificar el número de enlaces (del uno al diez) agrupados en estratos dicotómicos, indicada en la tabla 1.

\begin{tabular}{|l|l|}
\hline \multicolumn{2}{|l|}{$\begin{array}{l}\text { Enlaces variables de detección. ¿Cuántos enlaces tiene la } \\
\text { noticia? }\end{array}$} \\
\hline \multicolumn{1}{|c|}{ Código } & \multicolumn{1}{c|}{ Item } \\
\hline 0 & Ninguno \\
\hline 1 & $1-2$ \\
\hline 2 & $3-4$ \\
\hline 3 & $5-6$ \\
\hline 4 & $7-8$ \\
\hline 5 & $9-10$ \\
\hline 6 & 11 o más \\
\hline
\end{tabular}

Tabla 1. Códigos para el análisis de la detección de los enlaces. Fuente: elaboración propia a partir de Barrero (2003). 
6. Para calcular la calidad del uso del hipertexto se toma como referencia la metodología del ranking de calidad propuesto por Fondevila (2014) a través de un baremo cuantitativo de la hipertextualidad que permite medir su calidad según la cantidad de enlaces y que lo establece basándose "en algunas de las categorías propuestas por diversos autores (Díaz-Noci y Salaverría, 2003; Paul y Fiebich, 2003; Díaz-Noci, 2004; Salaverría, 2005) y en la escala de Likert (de 1 a 5)" (Fondevila, 2014, p.51). Este baremo se basa en cinco categorías numéricas objetivables para una aplicación empírica validadora: 5 (muy alta), 4 (alta), 3 (normal); 2 (baja); y 1 (muy baja), como se puede apreciar en tabla 2.

\begin{tabular}{|l|l|}
\hline \multicolumn{1}{|c|}{ Cantidad de enlaces } & \multicolumn{1}{c|}{ Valoración } \\
\hline$\geq 2$ por unidad de contenido & Muy alta (5) \\
\hline $1-1,99$ & Alta (4) \\
\hline $0,75-0,99$ & Normal (3) \\
\hline $0,25-0,74$ & Baja (2) \\
\hline $0-0,24$ & Muy baja (1) \\
\hline
\end{tabular}

Tabla 2. Baremo de hipertextualidad según calidad de enlaces utilizados. Fuente: Fondevila (2014).

7. Para identificar los tipos de enlaces que se usan en la divulgación científica se toma como base la propuesta metodológica para analizar los enlaces semánticos, según los contenidos, sugerida por Rost (2003, p. 179) conformado por cuatro categorías: de actualidad reciente (son enlaces a noticias del día); cronológicos (links que conducen a piezas informativas publicadas en fechas anteriores por el mismo medio); documentales (conducen a informaciones ampliatorias elaboradas por el medio a modo de grandes reportajes, o a las fuentes directa de la noticia en otros sitios de la web) e interactivos (conducen a foros, encuestas, etc.). Si bien, para nuestro estudio se mantienen los contenidos que componen las categorías "actualidad reciente" e "interactivos" pero se modifican los cronológicos (insertando también las piezas informativas de fechas anteriores publicadas por otros medios u organismos incluyendo cualquier género informativo como noticia, reportaje, entrevista, etc) y los documentales (que estarán conformados por toda aquella información que sea divergente a una pieza informativa).

Para computarlos se aporta una numeración a cada categoría para posteriormente procesarlos: 1 para actualidad reciente; 2 para cronológicos; 3 documentales y 4 interactivos. Y para describir cada una de las modalidades usadas en cada tipología, como se carece de metodología previa, se opta por agrupar las distintas modalidades que comparten las mismas características para concluir aportando una conceptualización a cada modalidad.

\section{Resultados}

Los resultados indican que del 100\% de las noticias publicadas en la Sección Ciencia en el periodo objeto de estudio, el 90,6\% de las piezas informativas están conformadas por enlaces (figura 1). Y dentro del cómputo de piezas informativas que tienen hipertexto, según el código para el análisis de la detección de enlaces, el número de éstos es, como se indica en la figura 2, por orden decreciente: $29,16 \%$ (entre 3 y 4 ); $25 \%$ (entre 1 y 2 ); 14,58\% (entre 5 y 6); 12,5\% (entre 7 y 8); 10,41\% (entre 11 y más); y $8,33 \%$ (entre 9 y 10 enlaces). Por tanto, la media del total de enlaces del periodo objeto de estudio $(n=245)$ es de 5,1 por pieza informativa. Una media que en el baremo cuantitativo establecido por Fondevila (2014) para medir la calidad de la hipertextualidad según la cantidad de enlaces usado es muy alta. Y no solo por la media, sino también porque dicho ranking, considera que "al menos debe incluir un enlace por unidad de contenido, de media" (Fondevila, 2014, p. 61) y en el caso objeto de estudio no sólo un $25 \%$ tiene entre 1 y 2 enlaces, sino que el

\section{Representatividad del uso de enlaces}

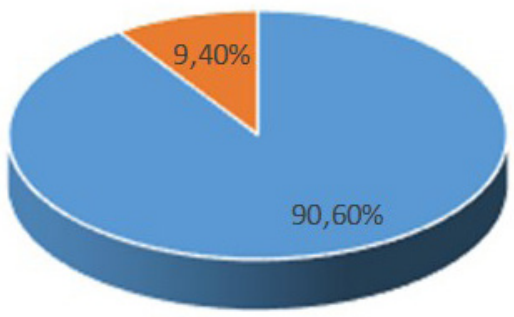

- Piezas informativas con enlaces

- Piezas informativas sin enlaces
Número de enlaces por pieza informativa

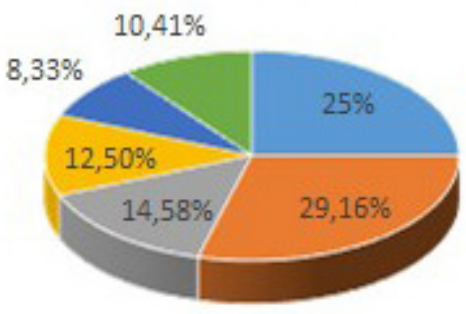

$$
\begin{array}{ll}
\text { - 1-2 enlaces } & \text { " 3-4 enlaces } \\
\text { = 5-6 enlaces } & \text { " 7-8 enlaces } \\
\text { - 9-10 enlaces } & \text { " } 11 \text { enlaceso má }
\end{array}
$$

Figuras 1 y $\mathbf{2}$. Representatividad del uso de enlaces y número de enlaces por pieza informativa. Elaboración propia. 
resto está por encima de esta media.

Datos que hacen interpretar que los hiperenlaces se consideran una herramienta eficaz para contextualizar y documentar los contenidos científico-tecnológicos, quizás porque permite al usuario decidir el grado de profundización de su lectura según su grado de interés o de formación, evitándole buscarlo en Google, al ofrecerle de manera escalada una gran cantidad de información y profundizar en el tema a su gusto. Y al periodista aumentar la calidad de los contenidos de todos los aspectos que considere aptos para enriquecer la lectura y también mostrar la importancia, veracidad o dudosa procedencia del hecho científico-técnico abordado. Al respecto, en una entrevista a Tascón en 2002, por Pérez Marco (2003) para su tesis doctoral, indicaba que el hipertexto es un texto enriquecido que al usarlo se hace pensando "que es el usuario quien elige cosas que hasta ahora sólo lo hacían los periodistas. El usuario ahora puede elegir la jerarquía informativa".

Unos hipervínculos, se destaca como dato significativo, que tienen una redirección directa y rápida para no distraer la lectura: el lector no tiene que clicar por segunda vez para acceder a la información aportada. En el 100\% de los casos la información del vínculo aparece con tan sólo un link.

Atendiendo a las tipologías de los enlaces los datos desvelan que no se hace uso de los enlaces de actualidad reciente ni interactivos en la pieza informativa (titular, lead y cuerpo de la noticia), centrado su uso en los cronológicos (58,3\%) y documentales (41,7\%). Y si bien, del cómputo total los datos indican que son más elevados los cronológicos sobre los documentales se destaca que en dos de las tres semanas analizadas son más elevados los documentales. Unos resultados que indican un uso combinado de ambos hipertextos.

Los de actualidad reciente, probablemente, no se usen porque en el medio digital las noticias se actualizan indicando la última hora de actualización, y no tiene sentido redireccionar a una misma noticia. Si bien, se considera que este enlace es de utilidad para redireccionar a otras piezas informativas publicadas sobre un acontecimiento el mismo día, pero que en la sección ciencia quizás no se use porque no se escriben diversas noticias sobre un mismo hecho, como puede ocurrir en los acontecimientos con conforman otras secciones. Y la ausencia de enlaces interactivos se interpreta que es debido a que se suelen insertar fuera del cuerpo de la pieza informativa (titular, lead y cuerpo de la noticia) que el objetivo de estudio de este análisis.

Los datos referentes al penúltimo y último de los objetivos, las modalidades que conforman cada una de las tipologías y cuáles son las más representativas desvelan que los enlaces documentales se usan para: mostrar los estudios científicos (redireccionando sólo al abstract, dejando al lector la decisión de descargar el documento, o bien, al artículo completo, ahorrándole dicha acción); a los perfiles académicos de los investigadores (en Twitter, Google Scholar, universidades o centros e institutos de investigación para los que trabajan o grupos de investigación a los que pertenecen; a libros (dirigiendo a la páginas de venta del libro o a la página exacta donde aparece la referencia hecha en la pieza informativa); informes (mostrándolos en su integridad); y a organismos científicos (museos, universidades, centros de investigación, institutos, academias, consejerías, organizaciones gubernamentales y no gubernamentales, fundaciones y empresas).

En concreto, los enlaces documentales más representativos por orden decreciente son: estudios científicos (33,7\%); organismos (30,7\%) y perfil del investigador/a (17,8\%). Por tanto, los más usados en primer lugar son los artículos científicos originales del que informan por la relevancia de su hallazgo en el medio de comunicación ofreciendo al usuario acceder a la fuente original, siendo más usuales las referencias sólo a los abstract $(69,7 \%)$ de dichos estudios que al estudio en completo en pdf (30,3\%), probablemente porque en los artículos científicos estos resúmenes están conformados por objetivos, metodologías, resultados y principales conclusiones que muestran una amplia referencia del mismo. $Y$ porque su lectura íntegra puede resultar dificultosa para un lector generalista, al estar escritos en un lenguaje científico técnico.

La segunda modalidad de enlace documental más usada son las referencias a las páginas webs de los organismos referenciados en la pieza informativa y que permite conocer la calidad de éstos. "Un enlace sencillo de incorporar y atractivo en periodismo digital" (Fondevila, Rom y Santana, 2016, p. 126). Y en tercer lugar a las páginas de los perfiles académicos de los investigadores. Un dato que se considera esencial en el periodismo y la divulgación científica porque si bien importa el que, más aún lo es el quién: para conocer la calidad de la fuente del hallazgo, o bien, la calidad de la fuente que lo contrasta. Y es que, por ejemplo, en el caso de los papers, aunque éstos son revisados por pares es preciso que el profesional de la información consulte con investigadores que trabajen en temas de la ciencia similares que puedan aportar sus comentarios al respecto. Y junto a estos datos se destaca que no se ofrecen comentarios opinativos sobre los perfiles profesionales ni sobre los organismos: la calidad de los mismos la decide el lector al visionar su currículum o la página web del organismo.

Los cronológicos, según los datos, se usan para redireccionar a piezas informativas para insertar piezas pretéritas. Y dichas informaciones se usan para: mostrar noticias que indiquen el hecho pasado del que hace referencia el texto principal; a series informativas, agrupadas a través de un cintillo, de todo lo publicado sobre un investigador/a o acontecimiento; a entrevistas o declaraciones del investigador/a dadas al medio; a reportajes que abordan la vida de un investigador o acontecimiento científico-tecnológico en profundidad; necrológicas y críticas de libros. Si bien, del 100\%, un 84,4\% son a piezas del propio medio y un $15,6 \%$ a otros medios de comu- 
nicación (BBC News, New York Times, Bruselas Times, Diario Pravda, El Tiempo), agencias (SINC, Reuters), al Consejo Superior de Investigaciones Científicas y Técnicas (CONICET) o la NASA, usados principalmente para indicar la procedencia de la fuente.

Sobre el elevado uso de enlaces internos al medio, Fondevila y Segura (2012) consideran que es "la constatación empírica de endogamia en los enlaces con el fin de que el lector no acuda a páginas de sus competidores" (p. 82). Si bien, en el periodismo y la divulgación científica se interpreta que es para ofrecer al lector mayor profundización sobre un acontecimiento previamente tratado por el medio, lo que se convierte en una pieza de relevante carácter documental y explicativa, que ahorra volver a explicar un hecho pasado. Al tiempo, que permite al usuario informarse de las primeras informaciones y posteriores publicadas sobre un hecho permitiéndoles conocer la sucesión de los acontecimientos desde sus orígenes. Una posibilidad de profundización y consulta de contenido anterior, que implica una transformación en los hábitos de trabajo hacia la reutilización de la información publicada anteriormente. En concreto, hacia el periodismo estructurado que parte "de historias que se codifican para servir de base a nuevas historias, y el material original de que se parte es periodístico [...] un procedimiento informativo en el que el sistema se retroalimenta periódicamente con nuevos datos" (Freixa, Pérez-Montero y Codina, 2017, p. 1078).

\section{Conclusiones}

La investigación pretende conocer el uso de los enlaces para divulgar la ciencia en el twitter del diario El País amateria-ciencia, la web de noticias de ciencia, tecnología, salud y medio ambiente. $Y$ los objetivos han sido conocer la media de enlaces insertados, las tipologías usadas, las modalidades que conforman cada tipología y las modalidades más representativas según tipología. Y las conclusiones obtenidas son las siguientes:

El diario El País, en este perfil de Twitter, hace un uso muy alto de los enlaces en las piezas informativas que informan sobre ciencia superando la media de un enlace, lo que implica una buena calidad en este recurso. Y las tipologías de enlaces más usados son los cronológicos y documentales, no tienden a usar los de actualidad reciente (piezas publicadas el mismo día por el medio u otros medios externos) o participativos (foros o encuestas) dentro de la pieza informativa (cuerpo, lead y texto). Esto implica que el profesional de la información hace un uso muy regular de este recurso documental lo que implica que se debe impartir cómo usarlos, en el sector académico a los futuros profesionales de la comunicación, como conductores para una lectura más profunda, detallada y referenciada del usuario. Una formación en la que sería muy relevante abordar la práctica de la curación de contenidos (Guallar, 2017) que hace referencia al tipo de contenidos abordados en el periodismo digital (información retrospectiva, reciente, actual o en tiempo real). Así como significativo es el decálogo ético de la curación de contenidos conformado por: citar a las fuentes, utilizar variedad de fuentes, y destacar a las fuentes.

Los enlaces documentales se usan en el periodismo científico principalmente para mostrar estudios científicos, redireccionar a la página web de los organismos y mostrar el perfil de los investigadores, junto a libros e informes. Y los cronológicos para mostrar principalmente noticias publicadas previamente por el medio. $Y$ estas modalidades obtenidas del estudio permiten establecer una primera clasificación, apta como herramienta académica y profesional, para elaborar piezas informativas sobre acontecimientos científicos:

Enlaces curriculares: datos académicos o perfil investigador del autor/a de una investigación o hallazgo científico. 0 bien, de los investigadores/as usados para contrastar o valorar dicho hecho. Un perfil que puede enlazarse a través de diversas modalidades: cuenta de Twitter, Google Scholar, universidades o centros e institutos de investigación para los que trabajan, grupos de investigación a los que pertenecen o dirigen, o directorios de expertos en los que están inscritos.

Enlaces organizacionales: conecta con la URL o dominio del organismo citado. Un enlace que conecta al lector con la página de inicio, o bien, con la pestaña específica donde está ubicada la información sobre la que se hace referencia en la pieza informativa.

Enlaces metodológicos-científicos: versión original del estudio científico que aporta un hallazgo publicado en una revista científica. Estudio del que se puede optar por insertar sólo el abstract o bien la descarga completa de la investigación.

Enlaces bibliográficos: referencias a libros que conectan con la editorial que vende el libro y que ofrecen entre otros datos, una sinopsis del mismo; o bien, a la página del libro digital donde está referenciada la cita expuesta en la pieza informativa.

Enlaces terminológicos: dirige al usuario a páginas, internas o externas del medio, que explican dicho concepto o teorías. Algunas modalidades son recurrir a videos de youtube o diccionarios terminológicos

Enlaces archivados: a piezas informativas escritas por un medio de comunicación (interno o externo) que informe, sin acotación temporal, del hecho al que se hace referencia en la pieza. Pueden versar sobre un acto pasado, las declaraciones vertidas por el investigador/a, o un reportaje, entre otras variables.

A partir de esta clasificación también se concluye con la propuesta de un modelo de construcción discursivo multilineal basado en la conexión de textos digitalizado para informar 
de los hallazgos científico-tecnológicos. Así, toda pieza informativa que aborde estos hechos debería indicar el perfil del investigador/a (enlace curricular), el del organismo al que pertenece (enlace organizacional) y la fuente original del estudio que permita consultar la metodología usada y la revista donde se ha publicado (enlace metodológico-científico). Así como, a la explicación de los términos, teorías, etc, que se consideren poco entendibles o que precisan de una explicación (enlaces terminológicos) y a referencias pasadas porque son un background que ayuda para conocer las causas consecuencias, los hechos pasados relacionados (enlaces archivados).

Estudios futuros ampliaran esta primera clasificación analizando el estudio del hipertexto en otros diarios nacionales de referencia. También se investigará en las revistas de divulgación científica con mayor tirada en España y se realizarán grupos de discusión con los responsables de redes o de las ediciones digitales. Y con el objeto de fomentar el modelo de construcción discursivo multilineal propuesto se pretenden organizar actividades de transferencia para impartirlo a profesionales en activo e insertarlo en las programaciones docentes sobre periodismo y comunicación científica.

\section{Referencias}

Arias-Robles, F., y García-Avilés, J. A. (2018). El destino del enlace periodístico: percepción de los editores y aplicación práctica de la hipertextualidad en los medios españoles. Palabra clave, 21(2), pp. 275-309. https://doi.org/10.5294/pacla.2018.21.2.2

Armentia, J. I., Caminos, J. M., Elexgaray, J., Marín, F., y Merchán, I. (2000a). El Diario Digital. Análisis de los contenidos textuales, aspectos formales y publicitarios. Barcelona: Bosch.

Armentia, J. I., Caminos, J. M., Elexgaray, J., y Merchán, J. (2000b) La información en la prensa digital: redacción, diseño y hábitos de lectura. Zer: Revista de estudios de comunicación, 8, 183-212. Recuperado de https://www.ehu.eus/ojs/index.php/Zer/article/ view/17422/15197

Barredo, D. (2003). Fuentes de información y enlaces hipertextuales en las alusiones al rey Juan Carlos en El País y ABC.es (2009-2011). Comunicación y Hombre, 89-113.

Belenguer, M. (2003). Información y divulgación científica: dos conceptos paralelos y complementarios en el periodismo científico. Estudios sobre el mensaje periodístico, 9, 43-53. Recuperado de https://revistas.ucm.es/index.php/ESMP/article/view/ ESMP0303110043A

Bernardino J.; Cebrián E. (2017). Función y valor documental de los enlaces semánticos en la prensa española (2002-2016). Miguel Hernández Communication Journal, n8, pp. 489- 519. http://dx.doi. org/10.21134/mhcj.v0i8.216

Borrras, L. (2005). Textualidades electrónicas: nuevos escenarios par la literatura. Barcelona: UOC.

Calvo Hernando, M. (1992). Periodismo Científico. Madrid: Paraninfo.

Castellanos, J. (2011). De lo impreso a lo digital. La migración de los periódicos impresos de América latina a los entornos digitales. Razón y Palabra, 77, 2. Recuperado de http://wwww.razonypalabra.org.mx/ varia/77\%202a\%20parte/23_Castellanos_V77.pdf
De Maeyer, J. (2012). The journalistic hyperlink. Prescriptive discourses about linking in online news. Journal Journalism Practice, 6(5-6), 692-701. https://doi.org/10.1080/17512786.2012.667273

Diazhandino, P. (1997). Especialización y periodismo de servicios, En F. Esteve, Estudios sobre información periodística especializada (pp. 83-90). Valencia: Fundación San Pablo CEU.

Díaz-Noci, J. (2018). Hipertexto en periodismo: realidad e investigaciones de futuro. Anuario ThinkEPI, v. 12, pp. 230-235. https://doi org/10.3145/thinkepi.2018.34

Estévez, F., y Fernández del Moral, J.F. (1997). Áreas de especialización periodística. Fragua: Madrid.

Fondevila, J. F. (2014). El uso del hipertexto, multimedia e interactividad en periodismo digital: propuesta metodológica de calidad, Zer, 19(36), 55-76. Recuperado de https://www.ehu.eus/ojs/index.php/ Zer/article/view/13488

Fondevila, J. F., Beriain, A., Del Olmo, J. L. y Carles, J. (2014). Interactividad, multimedia e hipertextualidad en el periodismo digital deportivo en España, En A.R Fernández, (Coord.) Interactividad y redes sociales, (pp. 231-244). Madrid: ACCI.

Fondevila J.F., Rom, J., y Santana, E. (2016). Comparativa internacional del uso de recursos digitales en el periodismo digital deportivo: estudio de caso de España y Francia. Revista Latina de Comunicación Social, 71, 124-140. http://dx.doi.org/10.4185/RLCS-2016-1087

Fondevila, J.F., y Segura Herlaynne (2012). La hipertextualidad en el periodismo digital en Colombia. Hipertext.net, 10. Recuperado de https://www.upf.edu/hipertextnet/numero-10/hipertextualidad-periodismo-digital-colombia.html

Fondevila, J., Berianin, A., Perelló, M., y Barbero, V. (2014). Estudio de caso de prensa digital internacional: hipertxtualidad, multimedia e interactividad en USA Today, Le Monde, The Telegrap y Clarín. En R. Colle, F. Campos, J.F. Fondevila, J. Bustos, J. Novoa (coords.), Estudios sobre la prensa digital iberoamericana (pp. 58-69). Alicante: Revista Mediterránea de Comunicación.

Freixa, P., Pérez-Montero, M., y Codina, L. (2017). Interacción y visualización de datos en el periodismo estructurado. El profesional de la información, v.26 (6), 1076-1090. https://doi.org/10.3145/epi.2017. nov.07

Galán, C. (2001). La ciencia como metáfora. Anuario de estudios filológicos XXVI, p. 123- 135.

García, M.A., Arévalo M.A., y Hernández, C.A. (2019). Estrategia de comprensión lectora: una propuesta para la lectura de los hipertextos. Saber, ciencia y libertad, 14(1). https://doi.org/10.18041/23823240/saber.2019v14n1.5230

Guallar, J. (2017). Artículos de curación de contenidos. Categorías y ejemplos. Anuario ThinkEPI, v. 11, 210-216. https://doi.org/10.3145/ thinkepi.2017.38

Herrero, C. (1997). Tratamiento y Estilos del Periodismo Especializado, en F. Esteve (coord..) Estudios de Información Periodística Especializada. Fundación Universitaria San Pablo CEU: Valencia.

Larrondo, A. (2009a). El reportaje hipermedia. Análisis del género en los especiales de elmundo.es, elpais.com y lavanguardia.es. Leioa: Servicio Editorial de la Universidad del País Vasco.

Larrondo, A. (2009b). La metamorfosis del reportaje en el ciberperiodismo: concepto y caracterización de un nuevo modelo narrativo. Comunicación y Sociedad, XXII(2), 59 - 88.

Larrondo, A. (2010) Propuesta metodológica para una aproximación 
empírica a los géneros ciberperiodísticos. Zer: Revista de estudios de comunicación, 15(28), 157-174. Recuperado de https://www.ehu.eus/ ojs/index.php/Zer/article/view/1648

Llano, S. (2005). Hipermedia e interactividad: teoría y práctica en los periódicos digitales colombianos. Palabra clave- Recuperado de http://palabraclave.unisabana.edu.co/index.php/palabraclave/ article/viewFile/1442/1575

López, X., Pereira, J., y Gago, M. (2001). ¿Llegó la hora del "lecto-periodista? Análisis de la utilización de enlaces externos en "Gaceta de Galicia". Estudios del mensaje periodístico, 7, 109-117.

Oblack, T. (2005). The lack of interactivity and hipertextuality in online media. The International Journal of Communication Studies, 67(1), 87-106.

Orero, P., Bernandino, J., y Cebrian, J. (2019): Evolución del uso del hipertexto en la prensa española (2002- 2016). El profesional de la información, 28(2). https://doi.org/10.3145/epi.2019.mar.25

Pérez, S. (2003). El concepto del hipertexto en el periodismo digital: análisis de la aplicación del hipertexto en la estructuración de las noticias de las ediciones digitales de tres periódicos españoles (wWW. elpais.es, www.elmundo.es, www.abc.es) [Tesis doctoral]. Universidad Complutense de Madrid.

Rodrigo, M. (1989). La construcción de la noticia. Madrid: Paidos.

Rost, A. (2003). Una propuesta metodológica para estudiar el hipertexto en el periódico digital. Anàlisi: Quaderns de comunicació $i$ cultura, 30, 169-183.

Rubio, M., y Blanco, J. C. (2010) Mejor que un buscador, un encontrador. Documentación de las Ciencias de la Información, 33, 27 -287.

Salaverría, R. (1999). De la pirámide invertida al hipertexto: hacia nuevos estándares de redacción para la prensa digital. Novatica, 142, $12-15$.

Steensen, S. (2011). Online journalism and the promises of new technology. Journalism Studies, 12(3), 311-327.

\section{CV}

Aida María de Vicente Domínguez. Doctora en comunicación por la Universidad de Granada. Especializada (máster) en comunicación científica, comunicación internacional y dirección de comunicación. Es profesora en los grados de Periodismo, Comunicación Audiovisual y Publicidad de la Universidad de Málaga. Una de sus principales líneas de investigación es la divulgación científico- técnica, principalmente centrada en la historia de las revistas de divulgación científica en el entorno digital (National Geographic, Muy Interesante y Quo), y la gestión de la información o del conocimiento científico-humanístico con el objeto de aportar recursos que permitan al profesional de la información acceder a fuentes fiables y documentar los hechos en el entorno digital. https:// www.directorioexit.info/ficha4983

Nereida Cea Esteruelas. Profesora de Periodismo en la Universidad de Málaga. Doctora en Ciencias de la Información (Periodismo) en la Universidad de Navarra. Máster en Periodismo Digital (Universidad del País Vasco) y Máster en Publicidad interactiva (Universidad Complutense). En el ámbito profesional ha trabajado en La Verdad (Grupo Vocento), La Economía (Grupo Intereconomía), El Mundo (Unidad Editorial). En el área de la comunicación política e institucional, responsable de comunicación de la Cámara de Comercio de Murcia y asesora en la Consejería de Presidencia de la Comunidad Autónoma de la Región de Murcia. https://www.directorioexit. info/ficha3245

\section{осм}

\section{Observatorio de Cibermedios}

\section{https://observatoriocibermedios.upf.edu/}
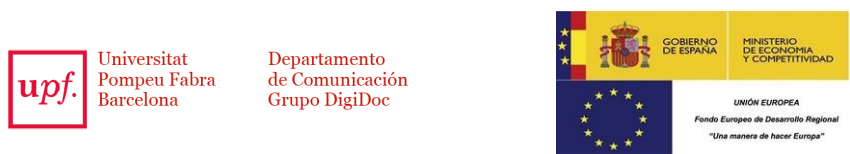

El Observatorio de Cibermedios es una producción del Grupo de Investigación en Documentación Digital y Comunicación Interactiva (DigiDoc) del Departamento de Comunicación de la Universitat Pompeu Fabra.

El Observatorio de Cibermedios (OCM) forma parte del proyecto del Plan Nacional "Creación y contenido interactivo en la comunicación de información audiovisual: audiencias, diseño, sistemas y formatos". CS02015-64955-C4-2-R (MINECO/ FEDER), Ministerio de Economía y Competitividad (España). 